\title{
Schluss: Wertvielfalt statt Wertfreiheit
}

The women's health movement, while a diverse movement, aimed to take our bodies back from the institutions of medicine and reframe our knowledge and experiences of our bodies in ways not configured by sexism and androcentrism. In this sense, the women's health movement was an epistemological resistance movement [...] an [...] important goal [...] was to reexamine traditional medicine, not simply in order to "get it right", but rather to transform our knowledge of women's bodies so as to remove oppression, to augment women's lives, and to transform society. (Tuana 2006, S. 2)

\section{Das Wertfreiheitsideal}

Ziel der Arbeit war die Beurteilung der Haltbarkeit und Fruchtbarkeit des Wertfreiheitsideals im Vergleich zu sozialerkenntnistheoretischen Alternativen, insbesondere Kitchers Well-Ordered Science und Longinos Social Value Management. Im Folgenden soll die Argumentation noch einmal zusammengefasst sowie auf die in Kapitel 2 formulierten Anforderungen an entsprechende Ideale (Kohärenz, Operationalisierbarkeit, Realisierbarkeit und Reichweite) bezogen werden. Eine Kritik des Wertfreiheitsideals sieht sich dabei zunächst vor die Frage gestellt, ob die Objektivität der Wissenschaft notwendig ihre Wertfreiheit voraussetzt. Diese Verknüpfung von Objektivität und (der Annäherung an) Wertfreiheit als unumgänglicher Anforderung an epistemische Qualität hat einen Großteil der bisherigen Diskussionen zu Werten in der Wissenschaft geprägt und erklärt den hohen Stellenwert, der dem Wertfreiheitsideal beigemessen wird. Frühere Kritiker der Vorstellung einer wertfreien Wissenschaft, beispielsweise das Strong Programme in der Soziologie wissenschaftlichen Wissens, haben entsprechend den Objektivitätsanspruch der Wissenschaft zurückgewiesen und diese als Politik mit anderen Mitteln zu entlarven versucht. Um die Möglichkeit einer Entkoppelung von Objektivität und Wertfreiheit - und damit einer fruchtbareren Diskussion - zu eröffnen, wurde zunächst ein Verständnis von Objektivität formuliert, das diese in der epistemischen Vertrauenswürdigkeit wissenschaftlicher Ergebnisse verortet, welche auf der Verwendung der bestmöglichen Prozesse bei der Produktion dieser Ergebnisse beruht. Durch eine solche prozedurale Auffassung von Objekti- 
vität, die von der Frage der Allgemeingültigkeit der erzielten Resultate abstrahiert, wird zunächst nicht impliziert, dass Werteinflüsse diese Vertrauenswürdigkeit zwingend untergraben.

Allerdings kann dies nicht bedeuten, dass von wertbeladenen Vorstellungen direkt auf die Gültigkeit wissenschaftlicher Aussagen über die Welt geschlossen wird, da nicht ersichtlich ist, wie auf dieser Grundlage epistemische Vertrauenswürdigkeit zu verteidigen wäre. Die Forderung nach empirischer Adäquatheit ist für eine solche unumgänglich und eine simple Ignoranz der vorliegenden Evidenz nicht hinzunehmen. Das Wertfreiheitsideal geht jedoch über diese minimale Version hinaus - wie auch die Möglichkeiten für Werteinflüsse in der Wissenschaft komplexer sind. Um dies zu verdeutlichen, wurde zunächst das Ideal der Wertfreiheit genauer spezifiziert und festgestellt, dass nicht alle Aspekte, die in seiner historischen Entwicklung von Bedeutung waren, in seiner aktuellen Version weiterhin enthalten sind. So fordert das heutige Ideal nicht mehr die Reinheit der Wissenschaft, d. h. dass die Themenwahl unabhängig von wertbeladenen Interessen sein müsse. An der Idee einer Neutralität der Wissenschaft wird zwar insofern festgehalten, als eine wissenschaftliche Begründung politischer Positionen abgelehnt wird; dennoch wird Wissenschaft nicht mehr als neutral im starken Sinn von ohne jede Verantwortung für die Konsequenzen ihrer Forschung betrachtet. Diese Verantwortung darf jedoch nach dem Wertfreiheitsideal die Beurteilung von Geltungsansprüchen nicht beeinflussen. Entscheidend für das Wertfreiheitsideal sind jene Aspekte, welche die Objektivität der Wissenschaft ermöglichen sollen: die Trennung von Sein und Sollen und der damit einhergehende Ausschluss von Sollensfragen sowie die Idee einer methodischen Kontrolle der Wissensproduktion. Dies entspricht der Unterscheidung des Rechtfertigungszusammenhangs von Entdeckungs- und Anwendungszusammenhang: Werteinflüsse auf die thematische Ausrichtung oder praktische Umsetzung sind für das gegenwärtige Wertfreiheitsideal unerheblich.

Das Wertfreiheitsideal zielt nicht auf eine völlige Unabhängigkeit oder Autonomie der Wissenschaft, sondern auf die Erfüllung der epistemischen Funktion, Objektivität zu ermöglichen. Dabei haben sowohl systematische Argumente als auch wissenschaftshistorische Untersuchungen zu der Einsicht geführt, dass die Beurteilung von Geltungsansprüchen nicht immer eindeutig und vor allem nicht immer allein durch Logik und Empi- 
rie vorgegeben ist. Das heutige Ideal zeichnet sich dadurch aus, dass es Werten eine wichtige Rolle bei der Theoriewahl zugesteht, da diese auf nicht-empirische Kriterien zurückgreifen muss. Mit diesem Zugeständnis geht jedoch die Forderung einher, wissenschaftliche (kognitive) und nichtwissenschaftliche (nicht-kognitive) Werte zu unterscheiden und nur erstere als Kriterien der Theoriewahl zu verwenden. Wertfreiheit bedeutet damit nun im Grunde Freiheit der Theoriewahl von nicht-kognitiven Werten.

Eine solche Auffassung macht zunächst die Unterscheidbarkeit einer Klasse kognitiver von nicht-kognitiven Werten zu einer notwendigen Bedingung von Wertfreiheit und damit (entsprechend der herkömmlichen Sichtweise) von Objektivität. Gleichzeitig wird (implizit) davon ausgegangen, dass diese Bedingung auch hinreichend für Wertfreiheit ist. Dies trifft jedoch nur im Zusammenhang mit zwei weiteren Annahmen zu: erstens, dass diese Trennung auch den Ausschluss nicht-kognitiver Werte ermöglicht; zweitens, dass die Rechtfertigung von Theorien von ihrem Entdeckungs- und Anwendungskontext epistemisch unabhängig ist.

Diese Fassung des Wertfreiheitsideals wurde in der Arbeit mit Bezug auf Beispiele aus der Frauengesundheitsforschung sowie auf philosophische Argumente für die Möglichkeit von Werteinflüssen kritisiert. Hinsichtlich der notwendigen Bedingung einer Trennbarkeit kognitiver und nicht-kognitiver Werte wurde argumentiert, dass die Möglichkeit einer kontextunabhängigen, absoluten Unterscheidung äußerst fraglich ist. Die Kognitivität von Werten wird über ihren Beitrag zu den Zielen der Wissenschaft bestimmt. Dabei ist sowohl strittig, was das Ziel der Wissenschaft ist als auch wie dessen Relation zu bestimmten Werten zu etablieren ist. Beispielsweise wurde die Annahme von Wahrheit als übergeordnetem Ziel und die entsprechende Bestimmung epistemischer Werte als wahrheitsfördernd dahingehend hinterfragt, wie diese Wahrheitsförderung zu begründen wäre. Dies ist insbesondere problematisch, da Werte wie Einfachheit oder Reichweite in keiner analytischen Beziehung zum Wahrheitsbegriff stehen und eine empirische Bestimmung des wahrheitsfördernden Beitrags die Möglichkeit voraussetzt, die Wahrheitsgrade verschiedener Theorien zu vergleichen. Wenn diese Wahrheit über empirische Adäquatheit und logische Konsistenz hinausgehen soll, was überhaupt erst die Grundlage des Problems bildet, sind für einen solchen Vergleich jedoch nicht-empirische Kriterien bereits vorauszusetzen. Auch bei anderen 
übergeordneten Zielvorstellungen wie etwa Verstehen bleibt - gerade aufgrund ihrer Allgemeinheit - unklar, wie aus diesen heraus spezifische Werte zu begründen wären.

Die fruchtbarere Strategie scheint daher zu sein, von spezifischeren Zielen in konkreten Forschungskontexten auszugehen. Ein engerer Fokus erleichtert die Bestimmung einzelner Werte als förderlich, indem er die Vergleichbarkeit der Förderung dieser Ziele durch verschiedene Werte erhöht. Dadurch eröffnet sich jedoch die Möglichkeit, dass diese Ziele auch durch wertbeladene Motive geprägt sind - wie etwa im Beispiel der Frauengesundheitsforschung das Ziel, adäquateres Wissen über die Gesundheit von Frauen zu generieren, mit dem feministischen Ziel einer gerechteren Gesellschaft verknüpft ist. Im Anschluss an Longino wurde argumentiert, dass derartige feministische Zielsetzungen zu spezifischen Werten der Theoriewahl führen können, die gleichzeitig auch den Wissenszuwachs in einer bestimmten Hinsicht fördern. So finden sich in der feministischen Gesundheitsforschung Werte als Merkmale guter Forschung wieder, die in anderen Kontexten keine oder doch eine weniger prominente Rolle spielen, etwa die Aufdeckung relationaler Komplexität und ontologischer Heterogenität, die Beachtung möglicher sozialer Folgen von Forschung oder auch die Reflexivität der Forscherinnen in Bezug auf ihre eigenen Werthaltungen.

Ebenso wie es feministische kognitive Werte geben kann, müssen andererseits die klassischen kognitiven Werte nicht zwingend rein kognitiv sein, sondern können in bestimmten Kontexten auch als androzentristische kognitive Werte fungieren - etwa, wenn der methodischen Einfachheit oder der Reichweite grundlegender Annahmen der Vorrang vor geschlechtssensiblen Resultaten eingeräumt und männliche Gesundheit als Standard behandelt wird. Durch den exklusiven Bezug auf die herkömmlichen Werte der Theoriewahl ist daher nicht garantiert, dass andere Werte eliminiert werden. Das ergibt sich bereits aus dem Umstand, dass diese Kriterien als Werte fungieren, also Entscheidungen zwar anleiten, aber nicht eindeutig bestimmen. Dies entspricht dem Problem der Kuhn-Unterbestimmtheit: die erforderliche Gewichtung und Präzisierung der Kuhnschen Werte kann durch andere Werte beeinflusst werden. Durch eine Trennung kognitiver und nicht-kognitiver Werte wäre also nicht zugleich impliziert, dass die Anwendung ersterer in der Theoriewahl einen Einfluss 
letzterer automatisch eliminiert. Gleichzeitig müssen jedoch kognitive Werte, die im Zusammenhang mit politisch relevanten Zielsetzungen stehen, nicht zwingend einen Verlust der epistemischen Vertrauenswürdigkeit bedeuten.

Wahrheit als Ziel der Wissenschaft anzunehmen ist darüber hinaus problematisch, weil Wissenschaft nicht indifferent nach allen möglichen Wahrheiten sucht, sondern nach signifikanten Wahrheiten, wie vor allem Kitcher herausstellt. Diese Signifikanz kann zwar epistemische Aspekte haben, etwa wenn es um die Implikationen verschiedener Fragen für den bisherigen Wissensbestand geht. Sie kann aber auch durch soziale oder anwendungsbezogene Werte beeinflusst sein. Selbst Ausrichtungsentscheidungen, die sich ausschließlich auf epistemische Signifikanz beziehen, können zudem frühere wertbeladene Signifikanzentscheidungen perpetuiieren, die den heutigen Wissensstand und damit auch gegenwärtige epistemische Möglichkeiten historisch geprägt haben. Diese Möglichkeit wertbeladener Ausrichtungs- und Signifikanzentscheidungen ist dabei auch dem Wertfreiheitsideal zufolge legitim - hat jedoch weitreichendere Konsequenzen, als von diesem eingeräumt wird.

Die Zuschreibung von Signifikanz beschränkt sich nicht auf die Auswahl von Themen, sondern wirkt sich auch auf die konkrete Bearbeitung von Fragestellungen aus. Hierbei spielt zunächst das Problem der holistischen Unterdeterminierung eine Rolle: Zwischen theoretischen Hypothesen und ihren empirischen Konsequenzen bestehen keine direkten Folgerungsbeziehungen. Deshalb ist oftmals nicht eindeutig, welches die entscheidenden Konsequenzen sind oder wie mit uneinheitlichen und widersprüchlichen empirischen Ergebnissen umzugehen ist. Einerseits ist es in manchen Fällen möglich, dieselben Daten als Unterstützung für rivalisierende Hypothesen anzuführen, wie etwa in der man-the-hunter/womenthe-gatherer Diskussion. Andererseits kann eine Hypothese durch einen Teil der vorhandenen Evidenz gestützt, durch einen anderen in Zweifel gezogen werden, wie etwa im Falle der unterschiedlichen epidemiologischen und klinischen Ergebnisse zur Prävention von KHK durch HET. In diesen Fällen sind daher weitere Annahmen und Entscheidungen nötig, um auf der Basis der Evidenz über die Evaluierung einer Hypothese zu entscheiden. Diese Annahmen können wertgeprägt sein, wenn etwa wertbeladene Sichtweisen über einen Gegenstandsbereich bestimmten Hypothesen 
eine hohe Plausibilität verleihen und deshalb an anderen Stellen des theoretischen Netzes Revisionen vorgenommen werden. Beispiele sind die Einführung der Annahme einer Unterschiedlichkeit verschiedener Darreichungsformen von HET oder die Bezweiflung der methodischen Sauberkeit der epidemiologischen Studien. Eine derartige Rolle wertbeladener Hintergrundannahmen ist im Sinne des Wertfreiheitsideals unzulässig, da hier nur wissenschaftliche Kriterien eine Rolle bei der Evaluation von Geltungsansprüchen spielen dürfen. Sicherlich ist ein hartnäckiges und wertgeprägtes Festhalten an Hypothesen mittels einer Einführung von adhoc-Maßnahmen auch kritisierbar. Es ist jedoch nicht ersichtlich, wie im Hinblick auf die Uneindeutigkeit der Relation von Hypothesen und empirischen Konsequenzen zu garantieren wäre, dass Annahmen über ihre jeweiligen Beziehungen nicht durch Werthaltungen und wertgeprägte Plausibilitätseinschätzungen beeinflusst sind.

Darüber hinaus nehmen wertbeladene Signifikanzentscheidungen Einfluss darauf, welche Hypothesen überhaupt aufgestellt, welche Daten erhoben und welche Theorien entwickelt und weiter verfolgt werden. Der Rechtfertigungskontext, sprich die Beurteilung von Geltungsansprüchen, ist jedoch nicht unabhängig davon, welche Daten zur Verfügung stehen und berücksichtigt werden müssen und welche rivalisierenden Theorien es gibt, die ihrerseits unter Umständen gut gestützt sind. Hierbei ist nicht auszuschließen, dass es blinde Flecken entweder in Bezug auf die Daten oder auf theoretische Erklärungen gibt, die eine gegebene Theorie in Zweifel ziehen würden, aber aufgrund wertbeladener Sichtweisen und Relevanzeinschätzungen bisher nicht in den Blick geraten sind. So generiert die feministische Sicht auf Frauengesundheit Ergebnisse, die für die bisherige Forschung problematisch sind, beispielsweise zu Erlebnissen sexualisierter Gewalt im Zusammenhang mit PMS oder zu der kulturellen Prägung menopausaler Beschwerden. Exemplarisch sind hierfür auch die Ergebnisse der WHI, deren Durchführung im Vorfeld als überflüssig kritisiert wurde, dann jedoch zu entscheidenen Änderungen in der Bewertung von HET führte. Die Wissenschaft kann nicht jeden Aspekt der Welt erfassen. Sie muss Selektionsleistungen vornehmen, und es ist nicht zu garantieren, dass diese Selektionen nicht bestimmte Bereiche aus wertbeladenen Gründen systematisch als irrelevant ausschließen oder schlicht übersehen. Dabei können wertgeprägte Signifikanzentscheidungen jedoch zu einer einseiti- 
gen Datenlage oder einem eingeschränkten Theorieangebot führen, dessen Bewertung im Lichte von Alternativen anders aussähe.

Die Rechtfertigung von Theorien ist deshalb von ihrem Entdeckungskontext nicht epistemisch unabhängig, wenn auch Aspekte der Entdeckung nicht direkt über Geltungsansprüche entscheiden. Des Weiteren können auch anwendungsbezogene Fragen und Werte Einfluss auf die Evaluierung nehmen. Das Problem induktiver Risiken von Fehlentscheidungen in Fällen mit vorhersehbaren sozialen Konsequenzen generiert ein Argument dafür, in diesen Fällen Werturteile über diese Konsequenzen in Fragen darüber, wie viel Evidenz für eine positive Evaluierung ausreicht, einzubeziehen. Zunächst einmal scheint es in ethischer Hinsicht wünschenswert, bei erheblichen Konsequenzen von Fehlentscheidungen höhere Standards zu fordern. Gleichzeitig gibt es nicht nur immer die Möglichkeit von Fehlern, sondern sowohl von falsch positiven wie von falsch negativen Ergebnissen. Das Verhältnis dieser Fehlerwahrscheinlichkeiten ist in Fällen mit vorhersehbaren Konsequenzen der jeweiligen Fehler abzuwägen, weshalb sehr hohe Standards, die falsch positive Ergebnisse auf Kosten falsch negativer vermeiden, nicht in jedem Falle sinnvoll erscheinen.

Induktive Risiken generieren jedoch nicht nur ein ethisches, sondern auch ein epistemologisches Argument gegen die Forderung nach einer Wertfreiheit der Evaluation. Werteinflüssen auf das Verhältnis der Fehlerwahrscheinlichkeiten lässt sich nur sehr bedingt durch die Forderung nach einem neutralen Verhältnis dieser Wahrscheinlichkeiten begegnen, welches sie in einem Gleichgewicht halten würde. Zunächst einmal scheint dies in epistemischer Hinsicht nicht immer sinnvoll, da Wissenschaft nicht einfach nach allen möglichen Wahrheiten, sondern nach signifikanten Wahrheiten sucht, wobei falsch positive oder falsch negative Ergebnisse nicht zwingend dieselbe Bedeutung hinsichtlich unseres Wissensbestands haben. Zudem spielt das Verhältnis der Fehlerwahrscheinlichkeiten nicht nur bei der endgültigen Entscheidung über eine Hypothese oder Theorie eine Rolle, sondern wird bereits vorher durch eine Reihe von methodologischen, interpretatorischen oder taxonomischen Entscheidungen beeinflusst. Diesbezüglich ist in vielen Fällen unklar, ob und wie sich hier ein Gleichgewicht überhaupt erreichen ließe, etwa wenn es um die Setzung eines Signifikanzlevels, die Auswahl von Kontrollgruppen oder die Einteilung von Krebsstadien geht. Die Rechtfertigung von Theorien ist damit auch 
von wertbeladenen Einstellungen zu ihrer Anwendung nicht in allen Fällen vollständig unabhängig, wobei diese Werteinflüsse nicht zwingend die epistemische Vertrauenswürdigkeit untergraben müssen - vor allem in Bezug auf Entscheidungen, die eine Gleichgewichtskonzeption der Fehlerwahrscheinlichkeiten gar nicht erlauben.

Die Annahme, die Rechtfertigung von Theorien sei von ihrer Entdeckung und Anwendung epistemisch unabhängig (bzw. könne und solle dies sein), ist letztlich nicht haltbar. Anwendungsbezogene Werte können Einfluss auf das Verhältnis von Fehlerwahrscheinlichkeiten nehmen, entdeckungsbezogene können sich auf Signifikanzzuschreibungen, Revisionsentscheidungen und die Einschätzung der Akzeptierbarkeit von Theorien auf der Basis wertgeprägter blinder Flecke auswirken. Die Kontextunterscheidung im Rückgang auf Reichenbach wird oftmals für die Verteidigung des Wertfreiheitsideals ins Feld geführt, ist jedoch selbst problematisch. Eine temporäre Interpretation dieser Unterscheidung ist unplausibel und begründet noch keine Aussagen über die Legitimität von Werteinflüssen in verschiedenen Phasen. Derartige Aussagen werden durch eine logische Interpretation wiederum garantiert; diese steht aber vor dem Problem, dass die Beurteilung von Theorien nicht rein logisch konstruierbar ist (und dies zudem auch eine Rolle kognitiver Werte in der Theoriewahl ausschließen würde). Dadurch wird auch die disziplinäre Version der Kontextunterscheidung in Zweifel gezogen, d. h. die Unabhängigkeit normativer Wissenschaftstheorie von empirischer Wissenschaftsforschung sowie auch die Beschränkung des philosophischen Gegenstands auf die Rechtfertigung unter Ausschluss von Aspekten der Theorieverfolgung und Anwendung. Die Kontextunterscheidung ist nur als schlanke Unterscheidung einer deskriptiven und normativen Perspektive auf Wissenschaft haltbar, die jedoch keine Aussagen über die Legitimität oder Illegitimität von Werten impliziert.

Letztlich ist daher die notwendige Bedingung der Trennbarkeit kognitiver und nicht-kognitiver Werte nicht zugleich hinreichend für Wertfreiheit. Vielmehr müsste das Wertfreiheitsideal um die Forderung einer Reinheit und einer starken Neutralität der Wissenschaft ergänzt werden. Ein Ideal, das etwa bei der Forschungsausrichtung Werte zulässt, aber davon unabhängig auf die Wertfreiheit der Rechtfertigung zielt, ist letztlich inkohärent, da Werte in der Entdeckung die Rechtfertigung indirekt beeinflus- 
sen. Weder eine Forderung nach der Vollständigkeit von Evidenz noch nach einer Ausrichtung von Signifikanzzuschreibungen an rein epistemischen Kriterien wie den Kuhnschen Werten bieten dabei eine erfolgreiche Verteidigung gegen die Wertbeladenheit von Entdeckung Signifikanz und ihre Implikationen. Zudem kann Wissenschaftlern nicht eine Verantwortung für eventuelle Folgen von Fehleinschätzungen zugesprochen und gleichzeitig gefordert werden, die Schwere dieser Folgen dürften sich in keiner Weise auf das Verhältnis von Fehlerwahrscheinlichkeiten auswirken, selbst wenn unklar ist, ob es hier überhaupt ein epistemisch neutrales oder richtiges Verhältnis geben kann.

Neben der internen Kohärenz ist auch die Realisierbarkeit des Wertfreiheitsideals fraglich, z. B. in Bezug auf die Möglichkeit, eine klare Unterscheidung zwischen legitimen und illegitimen Werten der Theoriewahl $\mathrm{zu}$ treffen. Seine Operationalisierbarkeit ist ebenfalls problematisch, d. h. die Identifizierung von Schritten zu seiner Erreichung und die Entscheidbarkeit einer entsprechenden Annäherung an dieses Ideal, da beispielsweise auch eine Unterscheidung kognitiver Werte nicht garantiert, dass durch deren Anwendung andere Werte eliminiert werden, das Wertfreiheitsideal hier jedoch keine weitergehenden Möglichkeiten der Kritik bereitstellt. Zumindest aber ist die Reichweite dieses Ideals erheblich eingeschränkt, da es nur auf Bereiche reiner Wissenschaft anwendbar wäre. Dabei ist allerdings fraglich, ob sich eine solche Reinheit überhaupt etablieren ließe; ob sie ethisch wünschenswert ist; und wie es um die Fruchtbarkeit eines Ideals bestellt ist, das den größten Teil der heutigen Forschung als epistemisch minderwertig auszeichnen müsste.

\section{Kitcher}

Das Kohärenzproblem des Wertfreiheitsideals betrifft auch Kitchers Ideal einer wohlgeordneten Wissenschaft. Gerade Kitcher unterstreicht die Wertbeladenheit der Ausrichtung von Forschung, von Signifikanzentscheidungen und spezifischen Zielen, die Einfluss auf Kriterien der Evaluation nehmen. Sein Beharren auf einer Wertfreiheit der Rechtfertigung beruht dabei vor allem auf einer Zurückweisung der Unterdeterminierungsthese anhand des Agnostizismusarguments, dass in Fällen uneindeutiger Evidenz 
auf weitere, entscheidende Ergebnisse zu warten sei. Dabei ist nicht klar, wie sich die Frage, welche Ergebnisse die Wahl entscheiden, eindeutig beantworten ließe und wie zu gewährleisten wäre, dass derartige Relevanzbeurteilungen wertfrei sind und nicht etwa aufgrund wertbeladener $\mathrm{Zu}$ schreibungen von Irrelevanz bestimmte Daten schlicht übersehen werden. Hier macht es wenig Sinn, für ein Warten auf das Vorliegen der vollständigen Evidenz zu plädieren, da Wissenschaft auch nach Kitcher immer (möglicherweise wertbeladene) Selektionsleistungen vornehmen muss und die Frage der Vollständigkeit deshalb kaum entscheidbar wäre. Zudem können Werte auch beispielsweise methodologische oder interpretatorische Entscheidungen - und damit indirekt die Evaluierung von Geltungsansprüchen - beeinflussen, wie in Bezug auf induktive Risiken erläutert wurde. Die Notwendigkeit dieser Entscheidungen wird auch durch weitere Evidenz nicht obsolet. Des Weiteren wurde argumentiert, dass Kitchers Hervorhebung der sozialen Ebene wissenschaftlicher Signifikanz und Zielsetzungen Einfluss auf die Kriterien der Theoriewahl nimmt, die schon deshalb nicht immer durch weitere Evidenz entscheidbar wäre.

Zudem ist WOS hinsichtlich seiner Operationalisierbarkeit problematisch, da es auf einen Abgleich der gegebenen Wissenschaft mit den Ergebnissen einer hypothetischen idealen Deliberation zielt. Hier bleibt unklar, wie ein solcher Abgleich überhaupt ermöglicht werden soll; es scheint daher ratsam, WOS auf einer prozeduralen Ebene zu operationalisieren und zu evaluieren, die sich auf die schrittweise Verwirklichung dieses Deliberationsprozesses bezieht. Wie dies genauer zu spezifizieren wäre und wie es um die Realisierbarkeit einer solchen Konzeption steht, sind dabei Fragen, die einerseits nach einer Auseinandersetzung mit politischen und demokratietheoretischen Themen und andererseits nach empirischer Forschung (etwa zu partizipativen Verfahren) verlangen. Ihre Beantwortung geht damit über den Rahmen der vorliegenden Arbeit weit hinaus. Wichtig ist an dieser Stelle jedoch, dass Kitcher die disziplinäre Beschränkung der Wissenschaftsphilosophie auf Fragen der Rechtfertigung aufbricht und auch den Entdeckungskontext systematisch in die normative Reflexion einbezieht. Damit macht er grundlegende Fragen der Beziehung von Wissenschaft und Gesellschaft zu signifikanten Themen (nicht nur der feministischen) Wissenschaftsphilosophie, wobei die Reichweite dieser Fragen sich auf den gesamten Bereich wissenschaftlicher Forschung erstreckt. 


\section{Longino}

Die Relevanz von Werten für die Rechtfertigung von Theorien erfordert ein Ideal, das die Wertfreiheit nicht nur um eine Demokratisierung von Entdeckung und Anwendung ergänzt, sondern auch ihre innerwissenschaftliche Bedeutung erfasst. Longinos Ideal einer pluralistischen und durch Diversität gekennzeichneten Forschungsgemeinschaft und der entsprechende soziale und prozedurale Begriff von Objektivität scheinen geeignet, die Komplexität möglicher Werteinflüsse zu erfassen und dennoch eine Grundlage für die Sicherung epistemischer Vertrauensbildung zu schaffen. Wie beschrieben, ist ihr Ansatz allerdings ebenfalls mit einem Kohärenzproblem behaftet, da ihre Erlaubnis eines epistemologischen Pluralismus, der auf eine Diversität und mögliche Inkommensurabilität von Beurteilungskriterien hinausläuft, ihrer Auffassung sozialer Objektivität als transformativer Kritik aus verschiedenen Perspektiven zuwiderläuft. Diesem Problem kann jedoch durch den Einschluss einer normativen Forderung nach wissenschaftlichem Konsens und der Ablehnung jeweils nur lokaler Gültigkeit begegnet werden. Ein solcher Konsens wird gerade bei einem Einbezug vieler Perspektiven kaum immer erreicht werden. Wichtig ist jedoch das Erfordernis eines Konsensstrebens, das sich auf die Ziele von Forschung, die Standards der Theoriewahl und die empirischen Ergebnisse erstreckt. Nur solch ein mehrstufiger und konsensorientierter kritischer Prozess bietet eine hinreichende Grundlage für epistemische Vertrauenswürdigkeit.

Longinos Bedingungen für soziale Objektivität stellen dabei gleichzeitig eine Möglichkeit der Operationalisierung dar, deren konzeptionelle Weiterentwicklung eine lohnende Aufgabe für die Wissenschaftstheorie ist. Eine genauere Ausformulierung und Prüfung der Realisierbarkeit von Kriterien wie etwa transformativer Kritik und geeigneter Prozesse für diese ist dabei wiederum nicht unabhängig von empirischer Forschung zu leisten. So ist beispielsweise in Bezug auf die Forderung nach einer Gleichheit intellektueller Autorität (bei gleicher Qualifizierung) nicht nur die formale Möglichkeit entscheidend, dass z. B. Frauen wissenschaftliche Karrieren verwirklichen können. Diskriminierungen finden auch auf subtilere Weisen statt, deren Beurteilung beispielsweise Forschung zu Publikations- und Zitationsverhalten, zu Einstellungskriterien oder zu kommunikativen Un- 
terschieden in Diskussionen erfordert (so werden Frauen häufiger und nach kürzerer Redezeit unterbrochen, müssen oftmals mehr Publikationen vorweisen, um in denselben Rang zu gelangen wie Männer usw.; vgl. z. B. Rolin 2002, Schiebinger 1999). Auch Longinos Ansatz einer internen Demokratisierung von Wissenschaft als epistemischer Ressource ist daher keinesfalls als unabhängig von dem weiteren gesellschaftlichen Kontext und komplexen politischen und regulatorischen Fragen $\mathrm{zu}$ sehen - eine Demokratisierung des Zugangs $\mathrm{zu}$ wissenschaftlicher Tätigkeit und der wissenschaftlichen Kommunikation setzt ein entsprechendes soziales Umfeld voraus.

Von den hier behandelten Idealen ist Longinos Ansatz, ergänzt um eine Konsensforderung, insgesamt am besten geeignet, die epistemische Funktion zu erfüllen, wissenschaftliche Integrität und Vertrauenswürdigkeit zu gewährleisten. Ihr kontextueller Empirismus muss nicht sämtliche Werteinflüsse als entweder irrelevant oder illegitim disqualifizieren und den Objektivitätsanspruch der Wissenschaft auf unhaltbaren Forderungen nach Wertfreiheit aufbauen. Zugleich bietet er fruchtbarere Möglichkeiten der Kritik, da er die Ebene der wissenschaftlichen Gemeinschaft wesentlich in die Diskussion einbezieht. So sind nicht zwingend alle Werteinflüsse mit schlechter Wissenschaft gleichzusetzen; wohl aber können einseitige und unhinterfragte Wertannahmen die Wissenschaft in einer problematischen Weise prägen. Derartige Einseitigkeiten zu vermeiden, ist natürlich auch im Sinne des Wertfreiheitsideals; SVM bietet aber zugleich einen Mechanismus, wie ihrer Möglichkeit zu begegnen ist, und muss die Diskussion von Werteinflüssen nicht auf eine Dichotomie guter oder schlechter Wissenschaft reduzieren.

Zudem beschränkt sich das Ideal eines kritischen Prozesses hier nicht auf bestimmte Aspekte wie die Theoriewahl oder die Ausrichtung von Forschung, sondern kann auf die Vielfalt von Entscheidungen angewandt werden, die Wissenschaft charakterisieren - welche Theorien weiter verfolgt, welche Hypothesen aufgestellt, welche Daten erhoben und welche methodischen Designs als angemessen beurteilt werden, wie uneinheitliche Ergebnisse zu interpretieren und was geeignete Kriterien der Theoriewahl sind, welchen Annahmen Plausibilität zugeschrieben wird, welchen Modellen heuristisches Potential usw. In Bezug auf all diese Entscheidungen scheint es erstrebenswert, sie aus vielfältigen Perspektiven zu diskutieren, 
da die Möglichkeit von Werteinflüssen immer gegeben ist und insbesondere geteilten Werten eine große Selbstverständlichkeit zukommen kann. Wertvielfalt scheint die beste Strategie, derartige Selbstverständlichkeiten sichtbar und hinterfragbar zu machen und gleichzeitig auch die Fülle von Entscheidungspunkten deutlicher hervortreten zu lassen.

Hinsichtlich der Reichweite dieses Ideals lässt sich daher zunächst konstatieren, dass die hier mögliche Ausweitung des wissenschaftstheoretischen Fokus angesichts der Problematik der Kontextunterscheidung äußerst begrüßenswert ist. Darüber hinaus spielen Werte zwar sicherlich in bestimmten Bereichen der Forschung, etwa in der Medizin, eine größere Rolle als vielleicht in der Physik. Dennoch ist es nicht der Fall, dass SVM nur auf sehr spezifische Beispiele wie etwa das der Frauengesundheitsforschung anwendbar wäre. Vielmehr beruht dieses Ideal auf generellen epistemologischen Argumenten, die auch über dieses Beispiel hinaus für weite Bereiche der wissenschaftlichen Forschung relevant sind.

\section{Frauengesundheitsforschung}

Longinos sozialer Objektivitätsbegriff bietet einen geeigneten Rahmen für die dargestellte Entwicklung der Frauengesundheitsforschung. Einerseits hat diese einseitige androzentristische und sexistische Werteinflüsse in der bisherigen Wissenschaft sichtbar gemacht und blinde Flecken auf der Landkarte der Forschung erschlossen. Andererseits ist diese Entwicklung nicht schlicht als eine Annäherung an Wertfreiheit zu verstehen. Zum einen ist auch die Frauengesundheitsforschung durch Werte geprägt, die zu alternativen Signifikanzzuschreibungen und theoretischen Erklärungen führen, aber auch feministische kognitive Werte als alternative Beurteilungskriterien auszeichnen. Zum anderen ist die herkömmliche medizinische Forschung nicht insgesamt als schlecht zu charakterisieren.

Zwar gibt es eine Reihe von Beispielen dafür, wie aufgrund von Werthaltungen zu Frauen wissenschaftliche Standards verletzt werden, etwa wenn an einer Psychogenität von Schwangerschaftsübelkeit festgehalten und die empirische Evidenz zu betreffenden Korrelationen ignoriert wird. Solche Fälle, in denen Werte Einfluss auf die Evaluation von Hypothesen nehmen, indem sie über das Kriterium empirischer Adäquat- 
heit gestellt werden, lassen sich durch SVM wie auch das Wertfreiheitsideal zurückweisen. Die Situation ist jedoch nicht immer so eindeutig. So war etwa der Ausschluss von Frauen aus klinischen Studien kein direkter Ausdruck von Misogynie, sondern auch dem Bemühen um einen Schutz vor teratogenen Schäden oder wie im Fall der KHK zum Teil der Altersverteilung dieser Erkrankung geschuldet. Im Fall der HET gab es vor der WHI eine Reihe epidemiologischer Belege für deren präventive Wirkung. Auf dieser Grundlage schienen wiederum bestimmte Hypothesen plausibel, wobei diese Plausibilität auch von Hintergrundannahmen beeinflusst war, welche reproduktive Funktionen als zentral für die Gesundheit von Frauen auszeichneten. Die Frauengesundheitsforschung hat solche wertbeladenen Hintergrundannahmen sichtbar gemacht, dadurch jedoch nicht unbedingt alle Werteinflüsse eliminiert. Vielmehr hat sie auf der Grundlage alternativer Werte zu neuen Sichtweisen und Ergebnissen geführt, welche die bisherige Forschung in Frage stellten. Die Entwicklung der Frauengesundheitsforschung lässt sich damit insgesamt besser als Fortschritt in Bezug auf Wertvielfalt statt auf Wertfreiheit verstehen.

\section{Wissenschaft und Gesellschaft}

Natürlich lässt auch ein solches Ideal, das auf Pluralismus statt Wertfreiheit setzt, Fragen offen. Zunächst einmal ist ein ständiger Diskurs von allen Teilnehmern bezüglich aller Entscheidungen sehr zeitintensiv und erfordert in seiner konkreten Ausgestaltung eine Abwägung hinsichtlich der Investition von Arbeitskraft. Darüber hinaus wurde in Frage gestellt, ob Longinos Ideal neben der epistemischen auch die politische Rolle des Wertfreiheitsideals übernehmen kann, wertgeprägte Annahmen, beispielsweise Vorurteile über soziale Gruppen, als ungerechtfertigt zurückzuweisen. Dazu lässt sich sagen, dass Longinos Zurückweisung des Wertfreiheitsideals nicht darauf hinausläuft, dass jede wertgeprägte Theorie auch anerkannt werden muss. Vielmehr müssen Theorien einen kritischen Prozess überstehen, der problematische Einseitigkeiten zu identifizieren und auszuräumen sucht. Eine weitere Frage ist dabei, welche Perspektiven überhaupt als relevant oder zulässig für diesen Prozess erachtet werden und ob nicht auch eine durch Diversität gekennzeichnete Gemeinschaft immer 
noch systematisch bestimmte Perspektiven ausschließen kann. So kritisiert etwa Kourany, dass die Zulassung verschiedener marginalisierter Gruppen in die Wissenschaft nicht die Gefahr ausräumt, dass deren wissenschaftliche Vertreter zu einem gewissen elitären Konsens neigen (vgl. Kourany 2008). Welche Perspektiven relevant sind, ist natürlich immer auch eine Ermessensfrage und lässt sich nicht absolut beantworten. Zudem sind der Diversität innerhalb der wissenschaftlichen Gemeinschaft offensichtlich bestimmte Grenzen gesetzt, da ihre Mitglieder, gleich welcher Ethnizität, welchen Geschlechts oder welcher sozialen Schicht immer einen hohen Bildungsgrad haben werden, was damit einhergehen kann, dass sie sich in ihren beruflichen Biografien sowie damit verbundenen Sozialisierungsprozessen ähneln. Darüber hinaus scheint die Forderung nach einer Diversität der wissenschaftlichen Gemeinschaft kaum zu erfüllen, ohne dass die Voraussetzung einer demokratischen und egalitären Gesellschaft gegeben ist.

Ein weiteres Problem wurde von Kitcher in Bezug auf die Freiheit der Theorieverfolgung und die Frage, ob eine pluralistische Wissenschaft ihrerseits zu einer demokratischeren Gesellschaft beiträgt, identifiziert. Er konstatiert politische Asymmetrien in Bezug auf die (öffentliche) Wahrnehmung von Forschung in wertbeladenen Bereichen. So würden etwa Studien zu Intelligenzunterschieden zwischen den Geschlechtern, die zu positiven Ergebnissen kämen, viel stärker rezipiert, während gegenteilige Ergebnisse den Glauben an eine Unterlegenheit der Frau kaum erschütterten. Deshalb ist zumindest fraglich, ob hier ein Pluralismus, der auch feministische Forscherinnen einbezieht, in Bezug auf die gesellschaftlichen Implikationen überhaupt etwas ausrichten kann. Gleichzeitig würde auch ein Ausschluss derartiger Fragen unter Umständen nur dem Argument Gewicht verleihen, dass die Wissenschaft aus politischen Gründen eben die Wahrheit dieser Vorurteile befürchte und zu verdecken suche (vgl. Kitcher 2001, Kap. 8). Darüber hinaus wirft Kitcher vor allem in seinem neuen Buch (2011) die Frage auf, wie sich eine Aufgabe des Wertfreiheitsideals auf die Autorität und gesellschaftliche Anerkennung der Wissenschaft auswirkt. Komplexere Modelle zu Werteinflüssen in der Wissenschaft könnten sich als problematisch in Bezug darauf erweisen, die epistemische Vertrauenswürdigkeit der Wissenschaft, gerade im Licht oftmals widersprüchlicher Expertenmeinungen, öffentlich zu untermauern. 
Dies sind komplexe und schwierige Fragen, die im Rahmen der Arbeit nicht mehr behandelt werden können. Sie sprechen dafür, die Betrachtung nicht auf die Frage des wissenschaftsinternen Pluralismus $\mathrm{zu}$ beschränken, sondern auch dessen Verbindungen zu dem weiteren sozialen und politischen Umfeld einzubeziehen (wie dies Kitcher stärker tut). So ist etwa die Entwicklung der Frauengesundheitsforschung gerade nicht nur ein Beispiel für die epistemische Fruchtbarkeit eines breiteren Zugangs zur Wissenschaft, sondern vielmehr zunächst eine explizite Laienbewegung gewesen. Deren wissenschaftlicher Einfluss wäre ohne politische Maßnahmen und institutionelle Bedingungen wie etwa die Verpflichtung zur Inklusion von Frauen in klinische Studien, die Berufung von Frauen in Entscheidungspositionen oder die Finanzierung großer Projekte wie der WHI nicht denkbar. Nicht nur muss die Entstehung und Beurteilung von Theorien im Kontext der wissenschaftlichen Gemeinschaft gedacht werden - auch in Bezug auf diese Gemeinschaft lassen sich nur schwer interne Fragen von ihrem gesellschaftlichen Kontext abgrenzen.

Es stellen sich damit eine Reihe von Anschlussfragen, die im Rahmen einer sozialen Erkenntnistheorie besser behandelt werden können als in einem traditionelleren Modell, das die vermeintliche Unabhängigkeit von sozialen Einflüssen zu einem Indikator epistemischer Qualität macht. Das Wertfreiheitsideal bietet keine fruchtbare Basis, den komplexen $\mathrm{Zu}$ sammenhängen von Wissenschaft und Gesellschaft nachzugehen, während eine sozialepistemologische Perspektive auch den sozialen, politischen, ökonomischen und institutionellen Rahmen von Wissenschaft in seiner wissenschaftstheoretischen Bedeutung reflektieren kann. Diese Reflexion muss sich dabei nicht auf die Rechtfertigung von Theorien beschränken, sondern kann die vielfältigen Entscheidungspunkte wissenschaftlicher Forschung deutlich machen und zudem eine normative mit einer empirischen Sichtweise auf Wissenschaft verbinden. Vor allem aber muss die Aufgabe des heutigen Wertfreiheitsideals nicht die Negierung des Objektivitätsanspruchs implizieren. Vielmehr bietet ein Leitbild von Wissenschaft als eines sozialen, prozeduralen und pluralistischen Prozesses wissenschaftlicher Konsensfindung sogar die bessere Grundlage für epistemische Vertrauenswürdigkeit. 